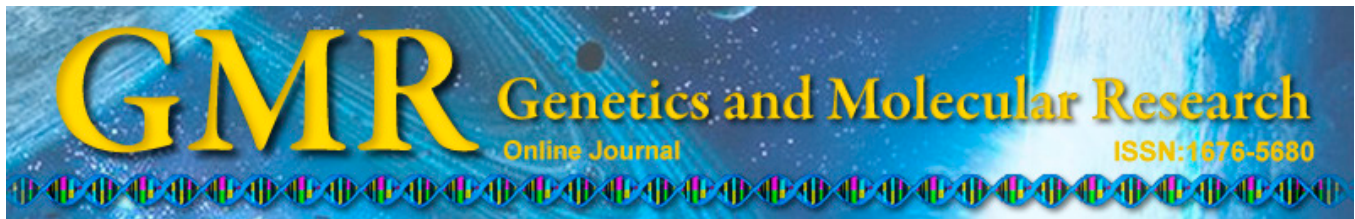

\title{
Impact of BMMSCs from different sources on proliferation of $\mathrm{CD}^{+} 4^{+}$cells
}

\author{
R.F. Liu, J.Q. Li, R.X. Hou, R. Wang and K.M. Zhang \\ Institute of Dermatology, Taiyuan City Centre Hospital, Taiyuan, China \\ Corresponding author: K.M. Zhang \\ E-mail: zhankm126@126.com
}

Genet. Mol. Res. 14 (1): 474-482 (2015)

Received July 2, 2014

Accepted November 21, 2014

Published January 23, 2015

DOI http://dx.doi.org/10.4238/2015.January.23.22

\begin{abstract}
There are significant differences on the biological characteristics of bone marrow mesenchymal stem cells (BMMSCs), immunological response, and antigen-presenting functions between patients with psoriasis and normal subjects, but there are no significant differences in aborted fetuses. We examined the differences in BMMSCs between aborted fetuses and patients with psoriasis in this study. Bone marrow from normal subjects, aborted fetuses, and patients with psoriasis were obtained using a MidiMACS machine. Density gradient centrifugation method was used to isolate the bone marrow mononuclear cells of patients with psoriasis and aborted fetus and the cells were cultivated. Bone marrow $\mathrm{CD} 34^{+}$cells from normal subjects were isolated. MTT colorimetric detection was used to test the proliferation activity of bone marrow $\mathrm{CD} 34^{+}$cells. The purity of bone marrow $\mathrm{CD} 34^{+}$cells and BMMSCs was determined by flow cytometry. The BMMSC culture supernatant fluid of patients with psoriasis and aborted fetuses showed no statistically significant difference with bone marrow $\mathrm{CD} 4^{+}$cell proliferation in normal subjects $(\mathrm{P}>0.05)$.
\end{abstract}

Key words: Bone marrow mesenchymal stem cells; $\mathrm{CD} 34^{+}$cells; Culture supernatant fluid; Proliferation activity; Psoriasis 


\section{INTRODUCTION}

Bone marrow mesenchymal stem cells (BMMSCs) are hematopoietic stem cells in the bone marrow, which acts as the microenvironment for their proliferation and differentiation (Jiang et al., 2002; Bonnet, 2003; Grove et al., 2004). Numerous studies have suggested that bone marrow cells are involved in the pathogenesis of psoriasis (Zhang et al., 2004; Niu et al., 2006; Yin et al., 2006). Our previous comparative studies revealed significant differences in the biological characteristics of BMMSCs (Zhang and Li, 2004; Zhang et al., 2010a,b), as well as the immunological response and antigen presenting functions between patients with psoriasis and normal subjects. However, we observed no significant differences in these features in aborted fetuses.

BMMSCs in the aborted fetus are found in the developmental stage, indicating that the immune status of BMMSCs in patients with psoriasis is significantly decreased and may be at the same level as in aborted fetuses. $\mathrm{CD}_{3} 4^{+}$is the surface marker of hematopoietic stem and progenitor cells. BMMSCs, another type of bone marrow stem cells, play an important role in the development and differentiation of $\mathrm{CD} 34^{+}$cells. Therefore, we examined the characteristics and differences in BMMSCs between aborted fetus and patients with psoriasis to provide a basis for follow-up studies. We cultivated BMMSCs from aborted fetus and patients with psoriasis to obtain the culture supernatant fluid and observed the impact of the culture supernatant fluid on the proliferation of bone marrow $\mathrm{CD} 34^{+}$cells in normal subjects.

\section{MATERIAL AND METHODS}

This cytology in vitro controlled study was conducted from July 2010 through April 2011 in the Dermatology Laboratory of Taiyuan Central Hospital.

Bone marrow was collected from normal subjects, aborted fetuses, and patients with psoriasis, and bone marrow cells were isolated using a MidiMACS machine (Miltenyi Biotec, Bergisch, Germany). All subjects provided informed consent.

\section{Samples}

The 9 aborted fetuses included in this study were aged 19-30 weeks, with an average of 25 weeks, and included 4 males and 5 females. The mothers were healthy and had voluntarily chosen to end the pregnancy using mifepristone and misoprostol. The placenta, umbilical cord, and fetus were preserved at a low temperature to prevent bacterial contamination. Consent was obtained from all donors.

Bone marrow samples were collected from patients with psoriasis who visited the Dermatological Department of Taiyuan Central Hospital and whose diagnosis was made using clinical and pathological methods. Nine patients were chosen including 5 males and 4 females, aged 24-49 years, with a mean age of 37.8 years, and disease duration was between several months and approximately 20 years. No subjects had used immunosuppressors, corticosteroids, vitamin A acid, or antibiotics. Antibiotics could not be used for 3 months before the study.

$$
\text { Bone marrow was from normal subjects in the Hematology Department. }
$$




\section{Experiment reagents and instruments}

CD34 ${ }^{+}$serum-free culture medium (Gibco, Grand Island, NY, USA), DMEM/F12 culture medium (Hyclone, Logan, UT, USA), fetal calf serum (Hyclone), stem cell factor, interleukin (IL)-3, HumanKine FLT3 ligand human (Sigma, St. Louis, MO, USA), lymphocyte separation medium (Tianjin Hao Yang Biological Manufacture Company, Tianjin, China), culture plates (Corning Inc., Corning, NY, USA), an inverted phase contrast microscope (Olympus, Tokyo, Japan), an EPICS-XL fluorescence microscopy (FCM) apparatus (Beckman Coulter, Brea, CA, USA), MidiMACS (Miltenyi Biotec), and clean bench (Suzhou Purification Equipment Company) were used in this study.

\section{Experimental methods}

\section{Separation and collection of bone marrow mononuclear cells (BMMCs)}

Bone marrow was collected from the 4 limbs of the fetus. Embryo marrow was processed using a previously described method (Zhang et al., 2009). Under aseptic conditions, we removed 4 long limb bones and used $0.9 \%$ normal saline to wash the bone marrow.

Next, $5 \mathrm{~mL}$ bone marrow samples were obtained from the posterior superior iliac spine of patients with psoriasis. Then, DMEM/F12 medium containing $10 \%$ fetal bovine serum was used to prepare a 1:2 dilution. Lymphocyte separation fluid density gradient centrifugation $(2000 \mathrm{~g}, 20 \mathrm{~min})$ was conducted and then the culture medium was washed once at $1000 \mathrm{rpm}$ for $8 \mathrm{~min}$ to isolate BMMCs. The supernatant was subjected to density gradient centrifugation to isolate the BMMCs from patients with psoriasis and aborted fetuses. The cells $\left(1 \times 10^{6}\right)$ $\mathrm{mL}$ ) were mixed with DMEM/F12 culture fluid containing $10 \%$ fetal bovine serum, $100 \mathrm{U} /$ $\mathrm{mL}$ penicillin, and $0.1 \mathrm{mg} / \mathrm{mL}$ streptomycin. Twenty-four-well culture plates were used, and the volume of each well was $1 \mathrm{~mL}$. The plates containing the culture supernatant were placed in a $\mathrm{CO}_{2}$ incubator at $37^{\circ} \mathrm{C}$ and $5 \%$ saturated humidity. After $72 \mathrm{~h}$, the culture medium in 1 well was subjected to FCM to determine the purity of BMMSCs. When the purity was more than $90 \%$, the culture supernatant fluid collected and centrifuged at $2000 \mathrm{rpm}$ for $15 \mathrm{~min}$. A $0.45-\mu \mathrm{m}$ Millipore filter was used to filter the samples from each well and the samples were stored at $-20^{\circ} \mathrm{C}$.

\section{Selection of $\mathrm{CD} 4^{+}$cells and identification using FCM}

The MidiMACS apparatus was used to separate and select CD34 ${ }^{+}$cells (Zhang et al., 2004). First, $5 \mathrm{~mL}$ bone marrow from the posterior superior iliac spine of normal subjects was used to prepare diluted samples in normal saline and was added to lymphocyte separation fluid at a ratio of 2:1. Density gradient centrifugation was conducted at room temperature at $2000 \mathrm{rpm}$ for $20 \mathrm{~min}$. The white membrane layer was carefully removed to fresh tubes and normal saline was used to wash the tubes, which were centrifuged again at $1000 \mathrm{rpm}$ for $8 \mathrm{~min}$. According to the instructions of the RBC pyrolysis liquid kit, the cell suspension was divided and $100 \mu \mathrm{L} 0.02 \%$ collagenase B was added. The tubes were shaken gently for $45 \mathrm{~min}$ at room temperature. The cells were passed over a $30-\mu \mathrm{m}$ nylon net and the supernatant was collected by centrifugation at $1000 \mathrm{rpm}$ for $8 \mathrm{~min}$. According to the 
requirements of the dead cell removal kit instructions, dead cells were removed from the cell suspension after the splitting of red blood cells; magnetic bead markers were added to $\mathrm{CD} 34^{+}$cells and MidiMACS was used to separate and select CD34 ${ }^{+}$cells. After selection, $100 \mu \mathrm{L}$ cells was removed for identification by FCM. Finally, the purity of CD34 $4^{+}$cells was found to be greater than $94 \%$.

\section{Culture of $\mathrm{CD}^{+} 4^{+}$cells under different culture conditions}

Sorted CD $34^{+}$cells were added to the BMMSC culture supernatant from patients with psoriasis and aborted fetuses for $24 \mathrm{~h}$. We used CD $34^{+}$cell serum-free medium containing $50 \mathrm{ng} / \mathrm{mL}$ stem cell factor, $20 \mathrm{ng} / \mathrm{mL}$ IL-3, $20 \mathrm{ng} / \mathrm{mL}$ FLT3 20, $100 \mathrm{U} / \mathrm{mL}$ penicillin, and $100 \mu \mathrm{g} / \mathrm{mL}$ streptomycin, and the cell density was adjusted to 5000 cells/well. Cells were inoculated on 96 -well culture plates at $100 \mu \mathrm{L}$ per well, to which $80 \mu \mathrm{L} \mathrm{CD} 34^{+}$cells serumfree medium containing cytokines are inoculated on the culture plates, and then 9 cases 20 $\mu \mathrm{L}$ spare BMMSC cultural supernatant of patients with psoriasis and aborted fetus were added respectively; each sample uses 3 wells (one original well and two duplicated wells). In addition, we selected additional six wells to added $\mathrm{CD} 34^{+}$cells with serum-free medium culturing in a humidified incubator at $37^{\circ} \mathrm{C}$ with $5 \% \mathrm{CO}_{2}$.

\section{Comparison of the proliferation of $\mathrm{CD} 34^{+}$cells under different culture conditions using MTT colorimetric assay}

An inverted microscope was used to observe the growth of cultured cells, including morphological changes and cell proliferation.

CD34 ${ }^{+}$cells were inoculated on a $96-$ well culture plate at 5000 cells $/$ well; to each well, we added $80 \mu \mathrm{L} \mathrm{CD} 34^{+}$cell serum-free medium containing $50 \mathrm{ng} / \mathrm{mL}$ stem cell factors, $20 \mathrm{mg} / \mathrm{mL} \mathrm{IL-3,} 20 \mathrm{ng} / \mathrm{mL}$ FLT3, and $20 \mu \mathrm{L}$ culture supernatant fluid. After $24 \mathrm{~h}$, the MTT colorimetric method was used to compare proliferation activity.

The purity of bone marrow CD $34^{+}$cells and BMMSCs was determined using FCM, while the proliferation activity of bone marrow $\mathrm{CD} 34^{+}$cells was detected using an MTT colorimetric assay.

The design of the experiment, data collection, implementation, and evaluation were conducted by the first author. Through systematic training, blinded evaluation was not used.

For statistical analysis, measurement data with normal distribution are reported as means \pm SD, determined using the $t$-test with the SPSS16.0 software (SPSS, Inc., Chicago, IL, USA). When $\alpha=0.05$ or $\mathrm{P}<0.05$, the data was considered to be statistically significant.

\section{RESULTS}

\section{Determination of $\mathrm{BMMSC}$ and $\mathrm{CD34}^{+}$cell purity using $\mathrm{FCM}$ after separation and selection}

In second-generation of BMMSCs, HLA-DR-FITC, CD45-PE, and CD34+-FITC were negative; CD29-PE and CD44-FITC were more than $90 \%$. CD34 $4^{+}$cell purity after separation and selection are shown in Figure 1. 

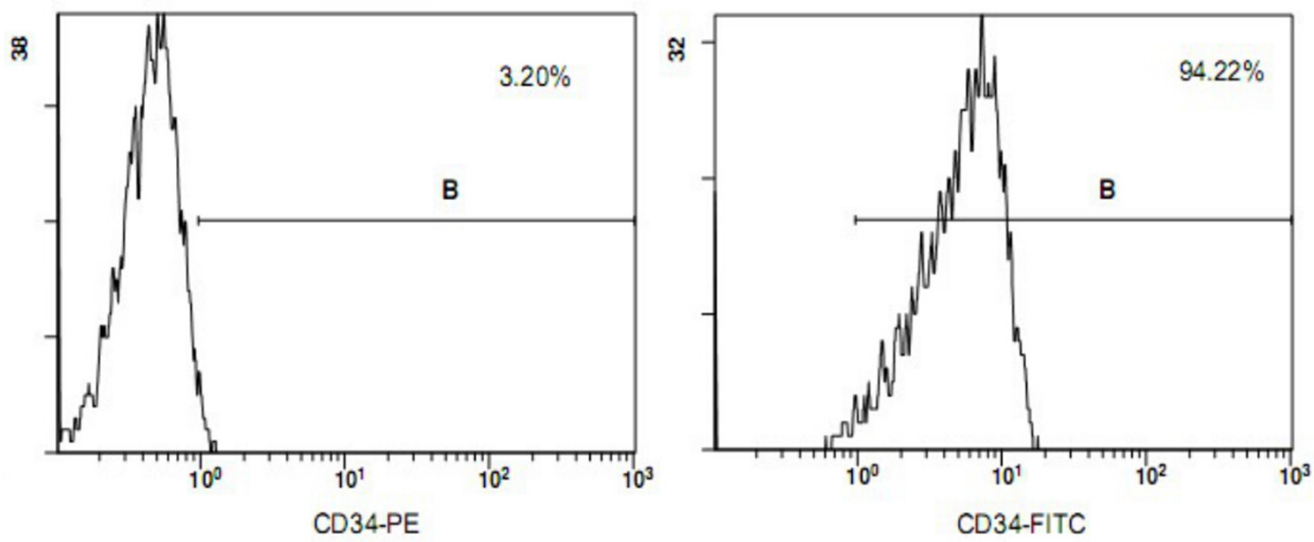

Figure 1. Results of $\mathrm{CD} 34^{+}$cells purity flow identification.

\section{Cell growth observed using an inverted microscope}

Using MidiMACS, we separated and selected $\mathrm{CD} 34^{+}$and added these cells to the BMMSC culture supernatant from patients with psoriasis and aborted fetuses. After inoculating the cells on 96-well culture plates, the $\mathrm{CD} 34^{+}$cells were round in shape (Figure 2). After $24 \mathrm{~h}$, the number of cells in the psoriasis patient group increased and showed growth; these cells were round (Figure 3). The same was observed in the aborted fetus group (Figure 4). The groups showed no significant difference under an inverted microscope.

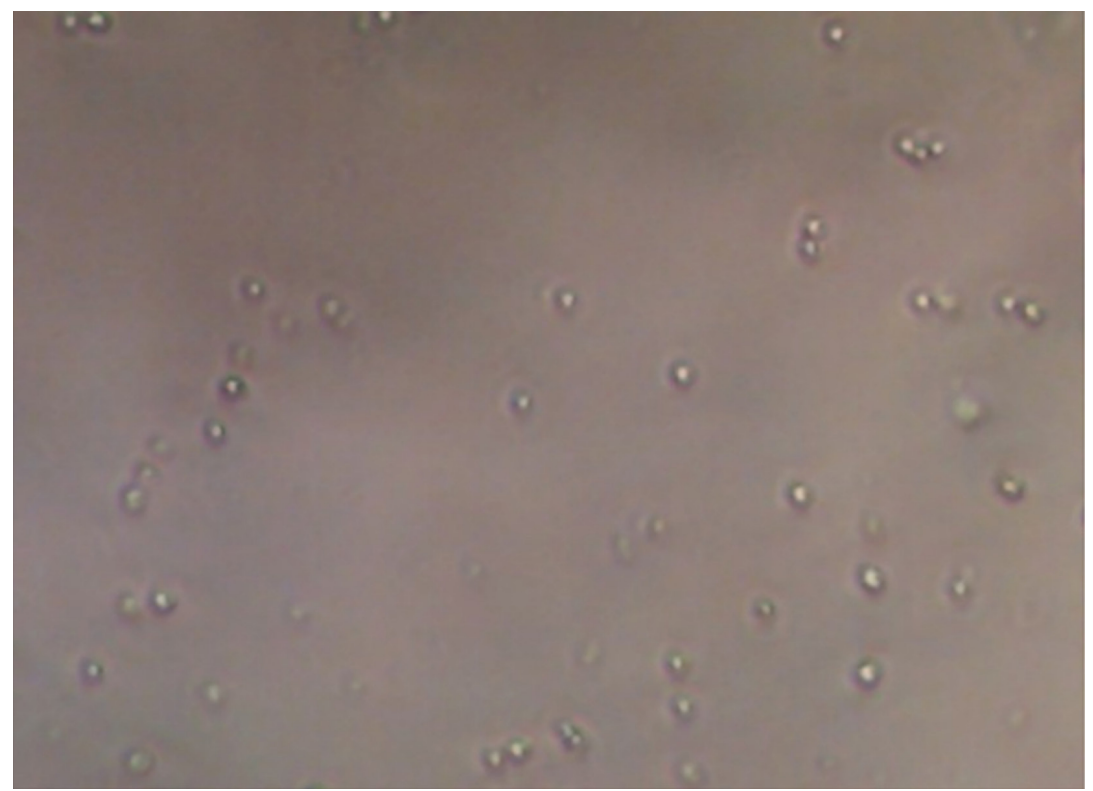

Figure 2. After separation and selection of $\mathrm{CD} 34^{+}$cells, added into serum-free medium (HP, 100X). 


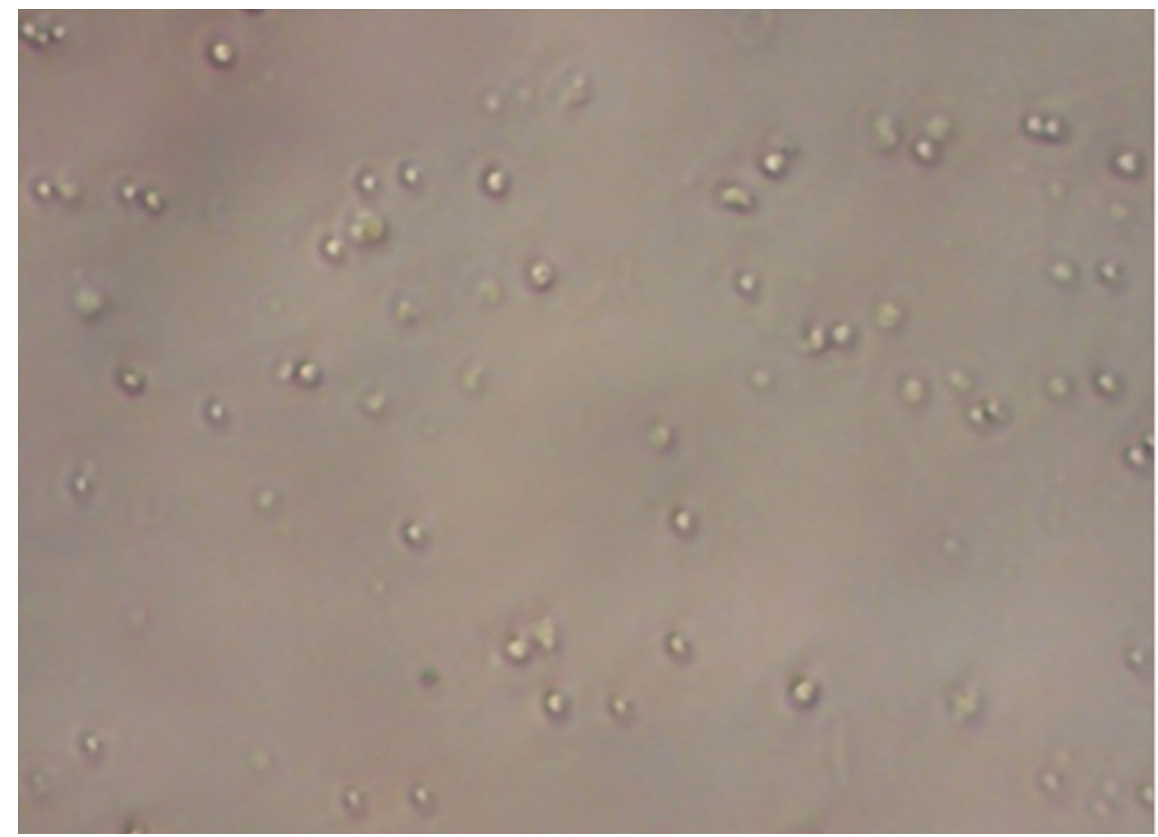

Figure 3. Addition of $\mathrm{CD} 34^{+}$cells to BMMSCs culture supernatant fluid of psoriasis patients group for $24 \mathrm{~h}$ (HP, 100X).

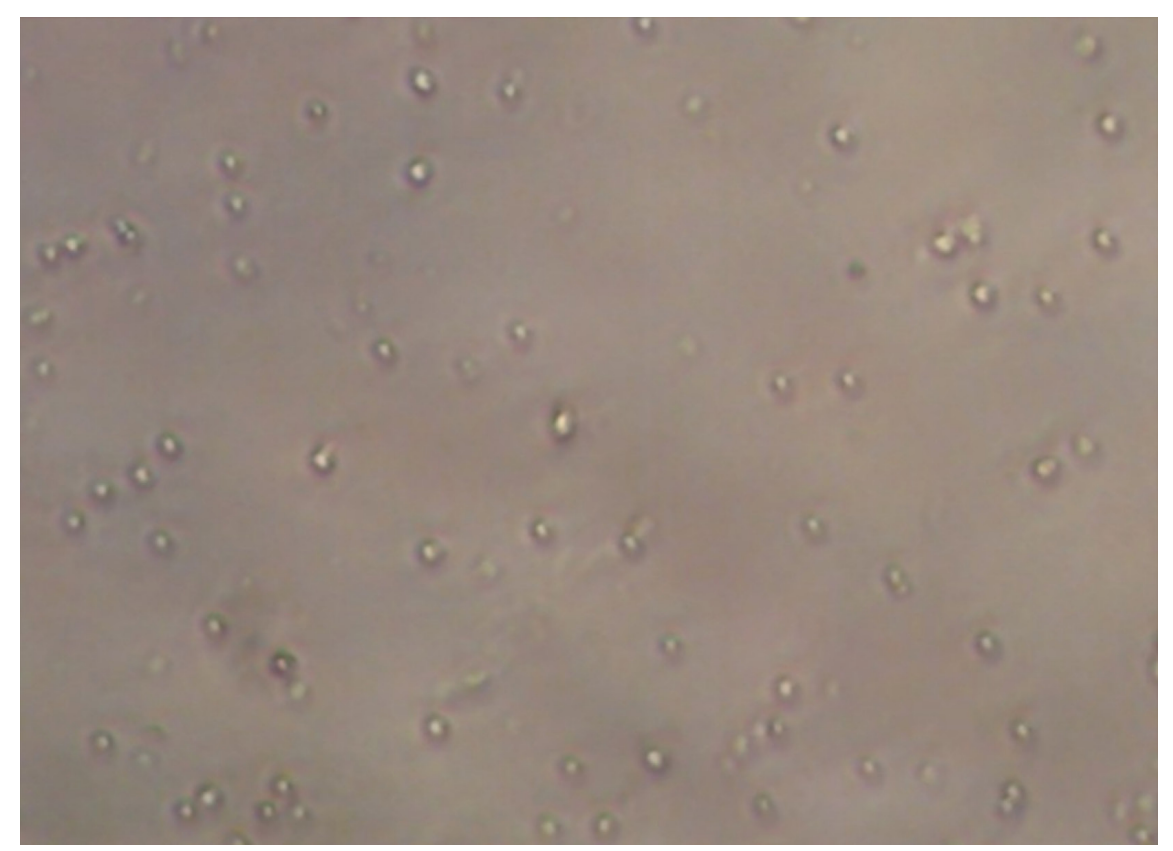

Figure 4. Addition of CD34+ cells to BMMSCs culture supernatant fluid of aborted fetus group for $24 \mathrm{~h}$ (HP, 100X). 


\section{Impact of BMMSC culture supernatant on proliferative activity of bone marrow $\mathrm{CD}^{+} 4^{+}$cells from normal subjects}

We added the BMMSC culture supernatant from patients with psoriasis and aborted fetuses to $\mathrm{CD} 34^{+}$cells serum-free medium for $24 \mathrm{~h}$ and found no statistically significant differences $(\mathrm{P}>0.05)$.

The absorbance values determined in the MTT colorimetric assay of cells from the psoriasis patient group and the control group are shown in Table 1.

Table 1. Proliferative activity.
\begin{tabular}{lcccc}
\hline Groups & Cases & Proliferative activity means \pm SD & $\mathrm{t}$ & $\mathrm{p}$ \\
\hline Psoriasis patients & 9 & $0.0453 \pm 0.0029$ & -0.258 & 0.80 \\
Aborted fetus & 9 & $0.0456 \pm 0.0035$ & & \\
\hline
\end{tabular}

\section{DISCUSSION}

Recent studies have indicated that hematopoietic cells play a key role in the pathogenesis of psoriasis (Li et al., 2007; Wan et al., 2009) and that patients with psoriasis have an abnormal hematopoietic microenvironment and CD34+ cells (Niu et al., 2007; Zhang et al., 2007, 2008; Li et al., 2009). BMMSCs are pluripotent cells that can be differentiated into bone cells, muscle cells, or endothelial cells, as well as other types of cells. These cells may be involved in immune responses, but may also secrete cytokines (Liu et al., 2009a,b), and may be involved in cell proliferation and differentiation, growth inhibition, apoptosis, chemotaxis, and voluntary movement (Wang et al., 2002; Ding et al., 2008; Toth et al., 2008; Yin et al., 2009). BMMSCs may also create a microenvironment for the proliferation and differentiation of bone marrow hematopoietic stem cells (Smart and Riley, 2008). A previous study showed that cytokines such as GM-CSF, IL-6, IL-7, IL-8, IL-11, IL-12, IL-14, and IL-15 secreted by BMMSCs can support the growth of hemopoietic progenitor cells (Deans and Moseley, 2000). We found no significant difference in the secretion level of GM-CSF, M-CSF, IL-7, and IL-11 in the psoriasis patient and aborted fetus groups as well as no differences in the immunological response and antigenpresenting functions. By observing the impact of BMMSCs on the proliferation of CD34+ cells in both groups, we found no significant difference, which initially confirmed the previous studies examining both groups, laying a foundation for further studies.

Recent studies have identified differences in BMMSCs from aborted fetuses and adults in supporting hematopoietic functions (Sun and Yang, 2009), suggesting that BMMSCs in aborted fetuses and adults may show different biological characteristics and immune responses. However, we found some similarities in BMMSCs from patients with psoriasis and aborted fetuses, as well as some differences in the level of cytokine secretion in patients with psoriasis and adults. Particularly the level of cytokine secretion of patients with psoriasis was similar to that of aborted fetuses. BMMSCs are important components of the hematopoietic microenvironment and play an important role in the proliferation and differentiation of hematopoietic stem cells (McNiece and Briddell, 2001; Wang and Wang, 2003; Jiang et al., 2008; Guo et al., 2009; Shi et al., 2010). Because BMMSCs in patients with psoriasis show abnormal activity, hematopoietic stem cells in psoriatic patients may exhibit abnormal activity. 


\title{
ACKNOWLEDGMENTS
}

\author{
Research supported by National Natural Science Foundation of China (\#81271768 \\ and \#81371736).
}

\section{REFERENCES}

Bonnet D (2003). Biology of human bone marrow stem cells. Clin. Exp. Med. 3: 140-149.

Deans RJ and Moseley AB (2000). Mesenchymal stem cells: biology and potential clinical uses. Exp. Hematol. 28: 875-884.

Ding G, Liu Y and Wang SL (2008). Immunological characteristics of mesenchymal stem cells. Bei Jing Kou Qiang Yi Xиe 16: 113-115.

Grove JE, Bruscia E and Krause DS (2004). Plasticity of bone marrow-derived stem cells. Stem Cells 22: 487-500.

Guo CJ, Hou D and Gao Y (2009). The cord blood hematopoietic stem/progenitor cells in vitro amplification preparations before and their clinical safety testing analysis. Zhe Jiang Da Xue Xue Bao 36: 328-334.

Jiang EL, Yang DL and Huang Y (2008). Between adult bone marrow mesenchymal stem cells on proliferation of hematopoietic stem cells in vitro. Sheng Wu Yi Xue Gong Cheng Yu Lin Chuang 12: 193-197.

Jiang Y, Jahagirdar BN, Reinhardt RL, Schwartz RE, et al. (2002). Pluripotency of mesenchymal stem cells derived from adult marrow. Nature 418: 41-49.

Li JQ, Li XH and Hou RX (2009). RUNX1 psoriatic patients bone marrow CD34+ cells and their target genes SLC9A3R1 research. Zhong Guo Mian Yi Xue Zha Zhi 25: 654-657.

Li X, Fan X, Zhang K, Yin G, et al. (2007). Influence of psoriatic peripheral blood CD4+ $\mathrm{T}$ and CD8 ${ }^{+} \mathrm{T}$ lymphocytes on C-myc, Bcl-xL and Ki67 gene expression in keratinocytes. Eur. J. Dermatol. 17: 392-396.

Liu RF, Yin GH and Zhang KM (2009a). Secretion of psoriatic patients with bone marrow stromal cells of stem cell factor and granulocyte colony stimulating factor levels. Zhong Guo Zu Zhi Gong Cheng Yan Jiu Yu Lin Chuang Kang Fu 13: 2713-2716.

Liu RF, Yin GH and Zhang KM (2009b). Psoriasis patients with bone marrow stromal cells secreted TNF alpha, LIF and HGF analysis. Zhong Guo Pi Fu Xing Bing Xue 23: 132-134.

McNiece I and Briddell R (2001). Ex vivo expansion of hematopoietic progenitor cells and mature cells. Exp. Hematol. 29: 3-11.

Niu XP, Xu HT and Yin GH (2006). Of psoriatic peripheral blood in patients with a single nuclear cell culture supernatant on bone marrow hematopoietic stem cells and progenitor cell colony formation. Zhong Hua Pi Fu Ke 39: 143-145.

Niu XP, Li XH and Zhang KM (2007). Psoriasis patients bone marrow hematopoietic cells in vitro proliferation activity research. Zhong Guo Pi Fu Xing Bing Xue 21: 196-198.

Shi XF, You HY and Ba Y (2010). Between bone marrow mesenchymal stem cells for hematopoietic stem cell "keep dry" function. Jiang Su Yi Yao 36: 1178-1180.

Smart N and Riley PR (2008). The stem cell movement. Circ. Res. 102: 1155-1168.

Sun XJ and Yang NL (2009). Adult and fetal bone marrow mesenchymal stem cells of biological character between comparison. Zhong Guo Zu Zhi Gong Cheng Yan Jiu Yu Lin Chuang Kang Fu 13: 2701-2704.

Toth ZE, Leker RR, Shahar T, Pastorino S, et al. (2008). The combination of granulocyte colony-stimulating factor and stem cell factor significantly increases the number of bone marrow-derived endothelial cells in brains of mice following cerebral ischemia. Blood 111: 5544-5552.

Wan YS, Li JQ and Zhang J (2009). CD34+ cells in patients with psoriasis and PKC beta I expression level detection. Chin. Skin Venereal Diseases 23: 132-134.

Wang JF and Wang LJ (2003). Bone marrow mesenchymal stem cells and hematopoietic stem cell co-culture between the umbilical cord blood hematopoietic stem cell amplification effect. Zhe Jiang Da Xue Xue Bao 30: 93-102.

Wang J, Luo CJ and Guo CH (2002). Mesenchymal stem cells and the related factors. Zhong Guo Shi Yan Xue Ye Xue 10: 468-471.

Yin GH, Li XH and Zhang KM (2006). Psoriasis patients bone marrow CD34+ cells in vitro directed differentiation of T cell activity research. Zhong Hua Pi Fu Ke 39: 124-127.

Yin GH, Liu RF and Zhang KM (2009). Psoriasis patients with bone marrow stromal cells IL-1 alpha, beta, and secretion of IL-11 IL-1 level of experimental research. Xian Dai Mian Yi Xue 29: 405-408.

Zhang KM and Li XH (2004). Bone marrow, psoriasis disease "center". Zhong Guo Pi Fu Xing Bing Xue 18: 501-503.

Zhang K, Zhang R, Li X, Yin G, et al. (2007). The mRNA expression and promoter methylation status of the p16 gene in colony-forming cells with high proliferative potential in patients with psoriasis. Clin. Exp. Dermatol. 32: 702-708. 
Zhang K, Li X, Yin G, Liu Y, et al. (2008). Functional characterization of $\mathrm{CD} 4{ }^{+} \mathrm{CD} 25^{+}$regulatory T cells differentiated in vitro from bone marrow-derived haematopoietic cells of psoriasis patients with a family history of the disorder. $\mathrm{Br}$. J. Dermatol. 158: 298-305.

Zhang K, Liu R, Yin G, Li X, et al. (2010a). Differential cytokine secretion of cultured bone marrow stromal cells from patients with psoriasis and healthy volunteers. Eur. J. Dermatol. 20: 49-53.

Zhang Y, Li LS and Fu XH (2004). Cord Blood CD34 cells in MACS sorting and significance. Hu Nan Shi Fan Da Xue Xиe Bao 1: 36-38.

Zhang YC, Feng HY and Liu RF (2010b). The expression of surface markers studied mesenchymal stem cells of bone marrow in patients with psoriasis. Zhong Guo Zhong Xi Yi Jie He Pi Fu Xing Bing Xue 9: 280-282.

Zhang ZY, Teoh SH, Chong MS, Schantz JT, et al. (2009). Superior osteogenic capacity for bone tissue engineering of fetal compared with perinatal and adult mesenchymal stem cells. Stem. Cells 27: 126-137. 